\title{
Retinal Venular Caliber Predicts Visual Outcome after Intravitreal Ranibizumab Injection Treatments for Neovascular AMD
}

\author{
Sanjeewa S. Wickremasinghe, ${ }^{1}$ Lucy Busija, ${ }^{1}$ Robyn H. Guymer, ${ }^{1}$ Tien Y. Wong, ${ }^{1,2}$ \\ and Salmaan Qureshi ${ }^{1}$
}

Purpose. To examine whether baseline retinal vascular caliber predicts visual response to intravitreal ranibizumab injections in patients with neovascular age-related macular degeneration (AMD).

Methods. In this prospective cohort study, patients with neovascular AMD received three monthly intravitreal injections of ranibizumab, followed by as needed dosing up to 1 year. Retinal vascular caliber was measured from digital fundus photographs at baseline and summarized as central retinal artery equivalent (CRAE) and venular equivalent (CRVE), representing average caliber of arterioles and venules, respectively. $\mathrm{Vi}$ sual outcome at 12 months was assessed and the relation to baseline retinal vascular caliber was determined.

REsults. A total of 88 eyes were analyzed at baseline. After accounting for age, sex, size of choroidal neovascularization, and number of injections, patients who deteriorated in visual acuity at 12 months had significantly larger baseline CRVE, 243.10 $\mu \mathrm{m}$ (95\% confidence interval [CI], 227.01-259.19), compared with those who were stable, $214.30 \mu \mathrm{m}$ (95\% CI, 205.79-222.81) and those who improved, $215.26 \mu \mathrm{m}$ (95\% CI, 204.69-225.84; $P=0.007)$. Baseline CRAE did not differ significantly from eyes whose vision deteriorated, $150.12 \mu \mathrm{m}$ (95\% CI, 140.67-159.57), compared with those remaining stable, $143.64 \mu \mathrm{m}$ (95\% CI, 138.64-148.63), or gaining vision $142.92 \mu \mathrm{m}$ (95\% CI, 136.71-149.13; $P=0.69$ ).

Conclusions. In eyes with neovascular AMD treated with intravitreal ranibizumab, larger baseline retinal venular caliber was significantly associated with a poorer response to treatment, possibly reflecting increased disease severity. (Invest Ophthalmol Vis Sci. 2012;53:37-41) DOI:10.1167/iovs.11-7689

A ntivascular endothelial growth factor (anti-VEGF) agents A have significantly improved the treatment of individuals with neovascular age-related macular degeneration (AMD). ${ }^{1,2}$

From the ${ }^{1}$ Centre for Eye Research Australia, Royal Victorian Eye and Ear Hospital Melbourne, University of Melbourne, Melbourne, Australia; and the ${ }^{2}$ Singapore Eye Research Institute, National University of Singapore, Singapore.

Supported in part by a Novartis fellowship (SSW), National Health and Medical Research Council Grant 52993, and Operational Infrastructure Support from the Victorian Government to the Centre for Eye Research Australia.

Submitted for publication April 5, 2011; revised September 1 and 28, 2011; accepted October 31, 2011.

Disclosure: S.S. Wickremasinghe, None; L. Busija, None; R.H. Guymer, Novartis (F, R, S), Pfizer (F, R, S); T.Y. Wong, Novartis (F, R, S), Pfizer (F, R, S); S. Qureshi, None

Corresponding author: Tien Y. Wong, Centre for Eye Research Australia, University of Melbourne, Royal Victorian Eye and Ear Hospital, 32 Gisborne Street, East Melbourne 3002, Australia; twong@unimelb.edu.au.
In neovascular AMD, choroidal neovascularization (CNV) has been associated with ischemic changes in the choroid, with increasing levels of ischemia correlating with severity of $\mathrm{AMD},{ }^{3,4}$ as well as an overexpression of vascular endothelial growth factor VEGF. ${ }^{5}$

Ranibizumab (Lucentis; Genentech, Inc., San Francisco, CA) is a recombinant, humanized, monoclonal antibody that neutralizes all isoforms of vascular endothelial growth factor-A, a key mediator of the neovascular process. ${ }^{3-7}$ The ANCHOR ${ }^{1}$ and MARINA ${ }^{2}$ studies demonstrated that ranibizumab was an effective treatment for neovascular AMD, and vision remained stable in approximately $90 \%$ and improved in approximately one third of treated patients. However, approximately $10 \%$ of treated patients continued to lose vision. ${ }^{1,2}$ Furthermore, trials assessing the efficacy of variable dosing regimens have generally shown a less favorable response, in terms of vision improvement compared with a monthly dosing regimen. ${ }^{8,9}$

Currently, it is unclear what factors predict response to treatment and identifying predictive factors would allow more precise methods to monitor, treat, and provide prognostic information to patients needing anti-VEGF treatment. Retinal vascular caliber is an indirect indicator of ocular blood flow that can be measured from fundus photographs. ${ }^{10}$ Changes in retinal vascular caliber have been linked with systemic and ocular diseases, and may reflect changes in retinal blood flow, inflammation, ischemia, and endothelial function. ${ }^{11,12}$ Thus, measurement of retinal vascular caliber may provide a marker of underlying disease severity in patients with neovascular AMD and may increase our understanding of which factors influence visual outcome in patients needing anti-VEGF treatment.

The aim of this study was to investigate the relationship between baseline retinal vascular caliber and visual response at 12 months, in eyes receiving anti-VEGF treatment by ranibizumab for neovascular AMD.

\section{Methods}

\section{Study Population}

This study was based on data from a prospective study evaluating the predictors of outcome of intravitreal ranibizumab on patients with CNV secondary to AMD. The study was approved by the research and ethics committee of the Royal Victorian Eye and Ear Hospital and followed the tenets of the Declaration of Helsinki.

Patients were recruited consecutively (January 1, 2008 to December 31, 2008) from the retina clinics of the Royal Victorian Eye and Ear Hospital, a tertiary eye care hospital in Melbourne, Australia. The inclusion criteria for this study were eyes with subfoveal/juxtafoveal CNV secondary to AMD that were deemed suitable for treatment with an anti-VEGF drug. There were no visual acuity exclusions, although 
the optical media needed to be clear enough to obtain sufficient quality photographs in the study eye.

\section{Study Eyes}

Over the 12-month recruitment period, all treatment naïve eyes with neovascular AMD were considered for inclusion, unless media opacity or mobility issues prevented adequate fundus visualization and in the view of the examiner would prevent adequate fundus photography.

\section{Measurement of Retinal Vascular Caliber}

All participants had fundus photography performed using a standardized protocol according to the Multi-Ethnic Study of Atherosclerosis. ${ }^{12}$ An optic disc-centered photograph was taken of each eye at baseline and at three monthly intervals in all participants (Early Treatment of Diabetic Retinopathy Study [ETDRS], Field 1) ${ }^{13}$ All photographs were taken before any treatment (TRC50 EX fundus camera; Topcon Corp., Tokyo, Japan). At baseline, photographs were taken within 48 hours of the first treatment. For each subsequent visit, photographs were taken before any treatment given, if required.

Retinal vascular caliber was measured by a masked grader, using a computer-based program (IVAN, University of Wisconsin, Madison, WI), according to an established protocol. ${ }^{11,14}$ In all cases due to the disc centration of photographs, the macula status was not visible to the grader. For each photograph (Fig. 1), all arterioles and venules coursing through an area one-half to one-disc diameter from the optic disc margin were measured and vessel calibers of the largest six were summarized as the central retinal artery equivalent (CRAE) and the central retinal vein equivalent (CRVE) using formulas developed by Hubbard et al. ${ }^{14}$ and later modified by Knudtson et al. ${ }^{15}$ These equivalents are projected calibers for the central retinal artery and vein. Intragrader reproducibility of retinal vascular measurements for their study was excellent (intraclass correlation coefficients for CRAE and CRVE were both $>0.98) .{ }^{15}$

\section{Visual Acuity}

Visual acuity was measured at baseline and at 12 months by examiners masked to vessel measurements and to the treatments administered

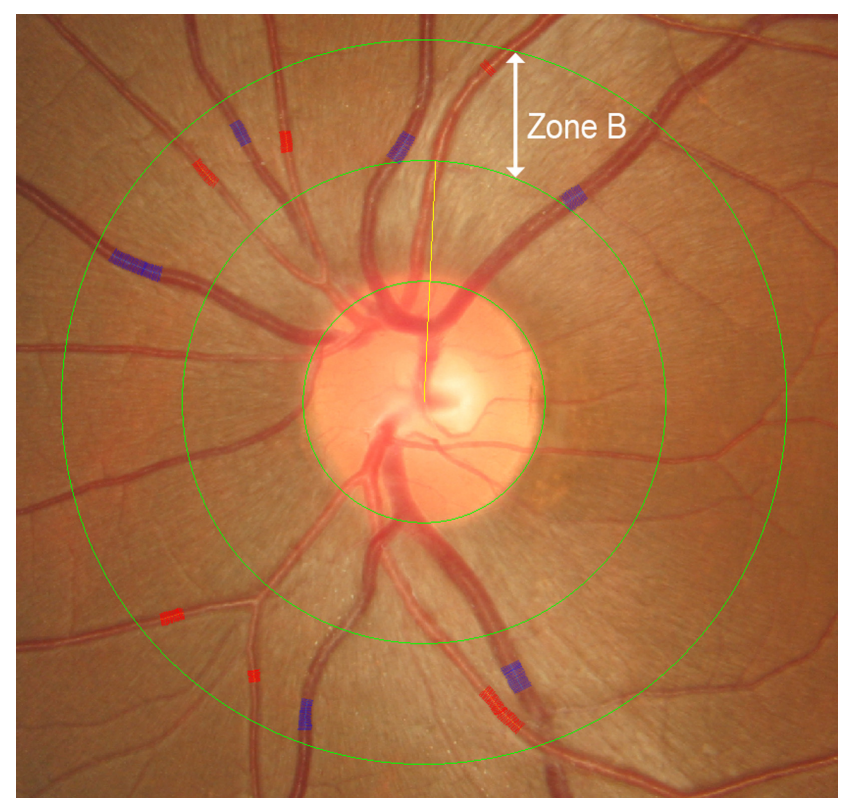

Figure 1. Disc-centered photograph of the left optic nerve head, highlighting the "Big 6" arterioles and venules used to calculate the central retinal artery equivalent (CRAE) and central retinal vein equivalent (CRVE). Change in central retinal vein equivalent with time in study and fellow eyes.
Best corrected logarithm of the minimal angle of resolution (logMAR) visual acuity was performed using ETDRS charts, after formal subjective refraction.

\section{Optical Coherence Tomography Acquisition and Analysis}

At baseline and at all subsequent visits, optical coherence tomography (OCT) was performed with OCT equipment (Stratus OCT 3; ZeissHumphrey Inc., San Leandro, CA), by an experienced technician, using the radial lines protocol. The protocol allowed qualitative scoring of the images for the presence of intraretinal fluid (edema/cysts), subretinal fluid as well as pigment epithelial detachments. A quantitative assessment of central macular thickness (CMT) was also made at each visit.

\section{Treatment with Ranizibumab}

After the initial "loading dose" regimen, further treatment was given on a pro re nata ("as needed") schedule based on regular clinical review, OCT findings, and patient symptoms. The retreatment criteria were the appearance of intraretinal or subretinal fluid on OCT or new retinal or subretinal bleeding. However, if continued loss of vision was observed, despite three consecutive monthly treatments and clinical features suggested irreversible retinal damage (fibrosis/atrophy), the decision to withhold treatment could be made by the treating specialist, although monthly assessment was continued up to 12 months.

The procedure for injection followed a standard protocol as previously described. ${ }^{16,17}$ In brief, eyes were prepared with several drops of amethocaine $1 \%$ and two drops of $5 \%$ povidone-iodine. A lid speculum was used in all cases. Lignocaine (2\%) was then administered subconjunctivally with a 30-gauge needle to the site of the intravitreal injection. Using a 30-gauge needle, $0.05 \mathrm{~mL}$ of ranibizumab $(0.5 \mathrm{mg}$; Novartis Pharmaceuticals, Sydney, Australia) was injected through the pars plana into the vitreous cavity. Chloramphenicol ointment was instilled at the end of the procedure and chloramphenicol drops were given to take home for 3 days.

\section{Statistical Methods}

The primary outcome measure in this study was change in visual acuity at 12 months after the start of anti-VEGF treatment for neovascular AMD. Improvement in visual acuity was defined as a two-line gain in vision (0.2 logMAR) at 12 months. Stable vision was defined as visual acuity within two lines of baseline during the same time period. Decrease was considered to be a reduction in visual acuity of two lines or more. The associations between primary outcomes and baseline characteristics of the study eyes were assessed using Pearson's $\chi^{2}$ for categorical variables and ANOVA or Kruskal-Wallis test, as appropriate, for continuous variables.

Multinomial logistic regression analysis was used to evaluate the ability of baseline vascular measurement categories to differentiate those whose visual acuity deteriorated from those who stayed stable or improved at 12 months, after controlling for other characteristics that were found to be significantly associated with the primary outcome in univariate analyses. Given the correlated nature of the data, the method of generalized estimating equations (GEE) was used to derive regression coefficients. All covariates were simultaneously included into the model. Associations between a predictor and the outcome were assessed using odds ratios (ORs) with 95\% confidence intervals (CIs). All statistical analyses were conducted using commercial software (SPSS version 18; SPSS, Inc., Chicago, IL). The nominal level of statistical significance was set at 0.05 .

\section{RESULTS}

Eighty-eight eyes had gradable photographs at baseline and 74 had photographs and information on visual acuity available at 12 months. At baseline, the majority of patients were females 
TABLE 1. Characteristics of Eyes Treated with Intravitreal Ranibizumab Injections for Neovascular Age-Related Macular Degeneration over the 12-Month Study Period

\begin{tabular}{|c|c|c|c|c|c|}
\hline Factor & Baseline & 3 Months & 6 Months & 9 Months & 12 Months \\
\hline Vision (LogMAR) & $0.73(0.40)$ & $0.60(0.40)$ & $0.66(0.46)$ & $0.71(0.48)$ & $0.70(0.50)$ \\
\hline CMT & $332(93)$ & $230(61)$ & $247(75)$ & $244(78)$ & $237(58)$ \\
\hline Residual fluid, \% & N/A & 31.6 & 36.4 & 33.6 & 20.9 \\
\hline Number of injections & N/A & $3.4(0.6)$ & $4.3(1.6)$ & $5.4(2.1)$ & $6.3(2.8)$ \\
\hline CRAE & $146.03(14.32)$ & $147.27(14.47)$ & $147.43(15.08)$ & $145.99(15.24)$ & $146.36(14.11)$ \\
\hline CRVE & $220.18(25.77)$ & $221.44(23.31)$ & $222.89(23.33)$ & $224.36(24.05)$ & $223.12(21.68)$ \\
\hline
\end{tabular}

Values are expressed as mean (SD) unless otherwise noted. Residual fluid indicates percentage of eyes with residual intraretinal or subretinal fluid based on qualitative assessment of optical coherence tomography scan, not applicable (N/A).

(64.2\%) and the mean age was 79.1 years (SD 7.9). Most lesions were classified as nonpredominantly classic (62.9\%) and were generally 2 disc diameters or less in size (71.3\%).

Table 1 shows the visual acuity and CMT changes through the 12-month follow-up period as well as the number of treatments given at each time point.

Despite the group level results (represented by mean scores) indicating no significant difference in visual acuity between baseline and final follow-up, a two-line change in vision occurred in several eyes over the study period, with $35 \%$ $(n=31)$ classified as having improved visual acuity and $20 \%$ $(n=18)$ classified as having worse visual acuity at 12 months compared with baseline.

\section{Patient Characteristics Affecting Vision at 12 Months}

The associations between patient/eye characteristics potentially influencing visual outcome at 12 months are summarized in Table 2. Sex, size of CNV lesion, and baseline CRVE were significantly associated with visual acuity outcome at 12 months $(P<0.05)$. Borderline associations were seen with baseline CMT, number of treatments given through to 12 months, and age of patient $(P<0.10)$. Eyes that deteriorated were found more frequently among males (56\%) and had higher frequency of large CNV lesions at baseline (53\%). After controlling for age, sex, and lesion size, baseline CMT, as well the number of treatments given, eyes that deteriorated had larger average venular caliber, $243.10 \mu \mathrm{m}$ (95\% CI, 227.01$259.19)$, than that of those who were stable, $214.30 \mu \mathrm{m}(95 \%$ CI, 205.79-222.81), and those who improved, $215.26 \mu \mathrm{m}$ (95\% CI, 204.69-225.84; $P=0.007$ ). Arteriolar caliber was not associated with visual outcome, with no significant difference between eyes losing vision, $150.12 \mu \mathrm{m}$ (95\% CI, 140.67159.57), compared with that of those remaining stable, 143.64 $\mu \mathrm{m}$ (95\% CI, 138.64-148.63) or gaining vision, $142.92 \mu \mathrm{m}$ (95\% CI, 136.71-149.13; $P=0.69$ ). No correlation between the baseline CMT and baseline CRVE $\left(r^{2}=0.01, P=0.33\right)$ or CRAE $\left(r^{2}=0.01, P=0.36\right)$ was noted. Similarly, CMT change

TABLE 2. Associations between Baseline Characteristics and Change in Visual Acuity at 12 Months, in Eyes Treated with Intravitreal Ranibizumab Injections for Neovascular Age-Related Macular Degeneration

\begin{tabular}{|c|c|c|c|c|}
\hline Factor & Better & Same & Worse & $P$ Value \\
\hline Sex, $n(\%)$ & & & & 0.03 \\
\hline Female & $18(58.1)$ & $32(78.0)$ & $8(44.4)$ & \\
\hline Male & $13(41.9)$ & $9(22.0)$ & $10(55.6)$ & \\
\hline Type of CNV, $n(\%)$ & & & & 0.06 \\
\hline NPC & $15(48.4)$ & $31(75.6)$ & $10(58.8)$ & \\
\hline PC & $16(51.6)$ & $10(24.4)$ & $7(41.2)$ & \\
\hline Size of CNV lesion, $n(\%)$ & & & & 0.02 \\
\hline$\leq 2$ disc areas & $26(86.7)$ & $28(68.3)$ & $7(46.7)$ & \\
\hline$>2$ disc areas & $4(13.3)$ & $13(31.7)$ & $8(53.3)$ & \\
\hline Hypertension, $n(\%)$ & & & & 0.62 \\
\hline No & $9(29.0)$ & $16(40.0)$ & $6(33.3)$ & \\
\hline Yes & $22(71.0)$ & $24(60.0)$ & $12(66.7)$ & \\
\hline Diabetes, $n(\%)$ & & & & 0.27 \\
\hline No & $28(93.3)$ & $33(82.5)$ & $14(77.8)$ & \\
\hline Yes & $2(6.7)$ & $7(17.5)$ & $4(22.2)$ & \\
\hline IHD/CVA, $n(\%)$ & & & & 0.94 \\
\hline No & $22(73.3)$ & $30(75.0)$ & $14(77.8)$ & \\
\hline Yes & $8(26.7)$ & $10(25.0)$ & $4(22.2)$ & \\
\hline Smoking, $n(\%)$ & & & & 0.16 \\
\hline Never smoked & $6(37.5)$ & $8(38.1)$ & $6(75.0)$ & \\
\hline Past or current smoker & $10(62.5)$ & $13(61.9)$ & $2(25.0)$ & \\
\hline Age, $y$, mean $(\mathrm{SD})$ & $75.65(7.45)$ & $78.59(7.37)$ & $80.72(7.83)$ & 0.06 \\
\hline Visual acuity at baseline, mean (SD) & $0.81(0.47)$ & $0.70(0.39)$ & $0.60(0.20)$ & 0.17 \\
\hline CRAE at baseline, mean (SD) & $144.32(14.52)$ & $143.90(15.73)$ & $148.23(11.07)$ & 0.44 \\
\hline CRVE at baseline, mean (SD) & $223.96(26.44)$ & $219.04(26.44)$ & $245.02(31.26)$ & 0.01 \\
\hline CMT at baseline, mean (SD) & $314.48(74.92)$ & $322.92(104.31)$ & $355.94(77.37)$ & 0.08 \\
\hline Number of injections at 12 mo, mean (SD) & $7.16(3.01)$ & $5.73(2.43)$ & $5.94(2.98)$ & 0.09 \\
\hline Axial length, mm, mean (SD) & $23.26(0.66)$ & $23.24(0.83)$ & $22.99(0.98)$ & 0.39 \\
\hline Spherical equivalent, D, mean (SD) & $-0.08(1.12)$ & $0.53(0.88)$ & $-0.38(2.93)$ & 0.83 \\
\hline
\end{tabular}

IHD, ischemic heart disease; CVA, cerebrovascular accident; NPC, nonpredominantly classic; PC, predominantly classic. 
from baseline to 12 months was not associated with either CRVE change $\left(r^{2}=0.03, P=0.20\right)$ or CRAE change $\left(r^{2}=0.03\right.$, $P=0.18)$. With respect to the treatment frequency, the mean number (range) of injections was 6.3 (3-13). In all, 19 eyes had four or fewer injections by 12 months. Of these the majority $(11 / 19)$ were considered as "fluid-free" after the three injections and were found to have either improved or stable vision by 12 months, compared with baseline. A further 5 eyes were "fluid-free" after three injections and had no recurrence of intraretinal or subretinal fluid through 12 months. These eyes, however, lost vision due to progression of atrophy or subretinal fibrosis and were, in the opinion of the treating ophthalmologist, unlikely to benefit from further treatment. The final 3 eyes had recalcitrant fluid through the 12-month follow-up and continued to lose vision despite the initial "loading dose" injections. These eyes were found to have significant subretinal fibrosis and further treatment was also considered as unlikely to influence vision.

Table 3 shows a GEE model assessing the combined effects of patient/eye characteristics and their influence on visual outcome at 12 months. Characteristics found to have marginally significant associations with the outcome were also initially included in the model because their addition changed the regression coefficients by $>5 \%$. By combining $\mathrm{CNV}$ size and type, however, the regression model produced singularity, possibly due to insufficient cell numbers. Consequently, only $\mathrm{CNV}$ size was included in the model, given that it had the stronger initial association with the outcome.

After adjusting for age, sex, baseline CMT, and number of injections received, only CNV size and baseline CRVE were able to differentiate those whose vision deteriorated at 12 months from those who stayed stable or improved. Eyes that had larger CRVE at baseline and larger CNV lesions had significantly lower odds of their vision improving or staying the same at 12-month follow-up.

With respect to the 14 eyes without images at 12 months, the reasons for lack of data included: failure of attendance or transfer to other ophthalmologists (11 eyes of 11 patients) and death of patients ( 3 eyes of 3 patients).

No significant differences $(P>0.05)$ were observed in age ( 78.5 vs. 79.4 years), sex ( $64.9 \%$ vs. $60.6 \%$ females), baseline vision (0.74 vs. 0.73$)$, baseline CRAE (144.6 $\mu \mathrm{m}$ vs. $147.8 \mu \mathrm{m})$, CRVE $(218.8 \mu \mathrm{m}$ vs. $221.9 \mu \mathrm{m})$, or CMT $(325 \mu \mathrm{m}$ vs. $348 \mu \mathrm{m})$ between the study completers and noncompleters.

\section{Discussion}

In this prospective study in eyes with active neovascular AMD treated with intravitreal ranibizumab injections, we found that baseline retinal venular caliber was a significant predictor of visual outcome at 12 months.

There are few studies that investigate the effect of ranibizumab on retinal vasculature. ${ }^{18,19}$ These studies were of small sample size (11 eyes of 11 patients), and found a decrease in retinal arteriolar diameter at both the 3-month time interval and 1 year after the initiation of treatment. No assessment was made of retinal venular caliber or any potential influence on visual outcome.

Factors such as patient age, sex, size of $\mathrm{CNV}$, and baseline vision have all previously been shown to influence visual outcome in patients with neovascular AMD. ${ }^{20,21}$ In addition to these factors, we found that patients who lost two or more lines of vision at 12 months had significantly larger venular diameters at baseline compared with those who were stable or those who had significant visual improvement. This finding remained significant even after adjusting for age and sex (both of which have previously been shown to affect retinal vascular caliber $^{22}$ ) as well as CNV size, baseline CMT, and number of injections given during the 12-month follow-up.

The specific underlying mechanism for our observations is unclear, but it is possible that the degree of retinal venular dilatation at diagnosis of $\mathrm{CNV}$ may be a marker of disease activity. In keeping with this hypothesis, retinal venular dilatation has been described as a predictive factor in the development of cardiovascular diseases such as stroke and coronary heart disease, independent of traditional cardiovascular risk factors. ${ }^{23-26}$ Additionally, retinal venular widening has been demonstrated in primates injected with vascular endothelial growth factor. ${ }^{27}$ As such, our observations of widening of retinal venules and poorer outcome of treatment with antiVEGF treatment are interesting and may represent either more severe disease at baseline, with increased VEGF levels in the eye or, alternatively, a marker of end-stage disease, with neural disorganization or fibrosis, predicting poorer response to antiVEGF therapy.

A weakness of our study was that only $80 \%$ of images were available at baseline at 12 months. We contend, however, that this is typical for this demographic of patients and no additional interventions were made (such as cataract surgery) purely to improve the data set. No significant differences were seen in CRAE, CRVE, and CMT in study eyes between those included in the analysis and those excluded at baseline. Accordingly, we feel that the general results were not substantially compromised. Another potential weakness of our study is the lack of standardized treatment protocol, with "as required" dosing allowed to maintain a "fluid-free" macula, rather than monthly dosing as seen in the pivotal randomized controlled

TABLE 3. Generalized Estimating Equations Modeling of the Effects of Baseline Characteristics on Visual Acuity Outcome at 12 Months in Eyes Treated with Intravitreal Ranibizumab for Neovascular Age-Related Macular Degeneration

\begin{tabular}{|c|c|c|c|c|c|c|}
\hline \multirow[b]{2}{*}{ Predictor } & \multicolumn{3}{|c|}{ Same Compared with Worse } & \multicolumn{3}{|c|}{ Better Compared with Worse } \\
\hline & OR & (95\% CI) & $P$ Value & OR & $(95 \% \mathrm{CI})$ & $P$ Value \\
\hline Female & Reference & & & & & \\
\hline Male & 0.50 & $(0.07-1.04)$ & 0.50 & 1.05 & $(0.11-9.54)$ & 0.97 \\
\hline$>2$ disc areas & 0.08 & $(0.01-0.59)$ & 0.01 & 0.02 & $(0.00-0.28)$ & $<0.001$ \\
\hline Number of injections at $12 \mathrm{mo}$ & 1.02 & $(0.74-1.40)$ & 0.92 & 1.18 & $(0.84-1.68)$ & 0.34 \\
\hline Central macular thickness & 1.00 & $(0.99-1.01)$ & 0.50 & 0.99 & $(0.98-1.00)$ & 0.25 \\
\hline
\end{tabular}

Same compared with worse (CNV): chance of vision remaining within two lines of baseline compared with losing vision by two or more lines. Better compared with same: chance of vision improving by two or more lines compared with remaining within two lines of baseline. 
trials. It is possible that monthly treatment may have influenced outcome, leading to better vision at 12 months. Finally, although the spherical equivalent of our patients did not differ significantly with respect to vision change at 12 months, it is possible that the axial magnification error due to refractive error may have influenced our results, although using a correction for refractive error based on the fundus camera (Zeiss [1 0.016] $\times$ spherical equivalent) ${ }^{28}$ our results were unchanged, such that after controlling for age, sex, CNV size, baseline CMT, and number of injections, eyes that had significant deterioration in vision at 12 months still had a larger CRVE (234.44 $\mu \mathrm{m}$; 95\% CI, 218.32-250.56), compared with that of eyes that were stable (210.27 $\mu \mathrm{m} ; 95 \% \mathrm{CI}, 200.94-219.60)$ and those that improved $(214.73 \mu \mathrm{m} ; 95 \% \mathrm{CI}, 204.88-224.56 ; P=0.04)$. This is consistent with our earlier work, indicating that the results are not affected by a potential bias due to axial magnification errors

In summary, our prospective study shows that in eyes with neovascular AMD, baseline retinal venular caliber is an independent predictor of visual response at 12 months and may be related to the severity of disease.

\section{References}

1. Brown DM, Kaiser PK, Michels M, et al. Ranibizumab versus verteporfin for neovascular age-related macular degeneration. $\mathrm{N} \mathrm{Engl}$ J Med. 2006;355:1432-1444.

2. Rosenfeld PJ, Brown DM, Heier JS, et al. Ranibizumab for neovascular age-related macular degeneration. $N$ Engl J Med. 2006;355: 1419-1431.

3. Lip PL, Blann AD, Hope-Ross M, Gibson JM, Lip GY. Age-related macular degeneration is associated with increased vascular endothelial growth factor, hemorheology and endothelial dysfunction. Ophthalmology. 2001;108:705-710.

4. Baffi J, Byrnes G, Chan CC, Csaky KG. Choroidal neovascularization in the rat induced by adenovirus mediated expression of vascular endothelial growth factor. Invest Ophthalmol Vis Sci. 2000;41:3582-3589.

5. Frank RN, Amin RH, Eliott D, Puklin JE, Abrams GW. Basic fibroblast growth factor and vascular endothelial growth factor are present in epiretinal and choroidal neovascular membranes. $A m J$ Opbthalmol. 1996;122:393-403.

6. Lopez PF, Sippy BD, Lambert HM, Thach AB, Hinton DR. Transdifferentiated retinal pigment epithelial cells are immunoreactive for vascular endothelial growth factor in surgically excised age-related macular degeneration-related choroidal neovascular membranes. Invest Ophthalmol Vis Sci. 1996;37:855-868.

7. Otani A, Takagi $\mathrm{H}$, Oh $\mathrm{H}$, et al. Vascular endothelial growth factor family and receptor expression in human choroidal neovascular membranes. Microvasc Res. 2002;64:162-169.

8. Regillo CD, Brown DM, Abraham P, et al. Randomized, doublemasked, sham-controlled trial of ranibizumab for neovascular agerelated macular degeneration: PIER Study year 1. Am J Ophthalmol. 2008;145:239-248.

9. Fung AE, Lalwani GA, Rosenfeld PJ, et al. An optical coherence tomography-guided, variable dosing regimen with intravitrea ranibizumab (Lucentis) for neovascular age-related macular degeneration. Am J Opbthalmol. 2007;143:566-583.

10. Sun C, Wang JJ, Mackey DA, Wong TY. Retinal vascular caliber: systemic, environmental, and genetic associations. Surv Opbthalmol. 2009;54:74-95.
11. Nguyen TT, Islam FM, Farouque HM, et al. Retinal vascular caliber and brachial flow-mediated dilation: the Multi-Ethnic Study of Atherosclerosis. Stroke. 41:1343-1348.

12. Wong TY, Islam FM, Klein R, et al. Retinal vascular caliber, cardiovascular risk factors, and inflammation: the Multi-Ethnic Study of Atherosclerosis (MESA). Invest Opbthalmol Vis Sci. 2006;47: 2341-2350.

13. Early Treatment Diabetic Retinopathy Study Research Group Grading diabetic retinopathy from stereoscopic color fundus photographs - an extension of the modified Airlie House classification. ETDRS report number 10. Ophthalmology. 1991;98(suppl 5):786806.

14. Hubbard LD, Brothers RJ, King WN, et al. Methods for evaluation of retinal microvascular abnormalities associated with hypertension/sclerosis in the Atherosclerosis Risk in Communities Study. Ophthalmology. 1999;106:2269-2280.

15. Knudtson MD, Lee KE, Hubbard LD, Wong TY, Klein R, Klein BE. Revised formulas for summarizing retinal vessel diameters. Curr Eye Res. 2003;27:143-149.

16. Aiello LP, Brucker AJ, Chang S, et al. Evolving guidelines for intravitreous injections. Retina. 2004;24(suppl 5):S3-S19.

17. Bylsma G, Guymer R, Qureshi S, et al. Intravitreous injections. Clin Exp Ophthalmol. 2006;34:388-390.

18. Mendrinos E, Mangioris G, Papadopoulou D, Donati G, Pournaras C. One year results of the effect of intravitreal ranibizumab on the retinal arteriolar diameter in patients with neovascular age-related macular degeneration. Invest Ophthalmol Vis Sci. 2011 Dec 13. [Epub ahead of print].

19. Papadopoulou DN, Mendrinos E, Mangioris G, Donati G, Pournaras CJ. Intravitreal ranibizumab may induce retinal arteriolar vasoconstriction in patients with neovascular age-related macular degeneration. Opbthalmology. 2009;116:1755-1761.

20. Kaiser PK, Brown DM, Zhang K, et al. Ranibizumab for predominantly classic neovascular age-related macular degeneration: subgroup analysis of first-year ANCHOR results. Am J Ophthalmol. 2007; 144:850-857.

21. Boyer DS, Antoszyk AN, Awh CC, Bhisitkul RB, Shapiro H, Acharya NR. Subgroup analysis of the MARINA study of ranibizumab in neovascular age-related macular degeneration. Opbthalmology. 2007; 114:246-252.

22. Wong TY, Kamineni A, Klein R, et al. Quantitative retinal venular caliber and risk of cardiovascular disease in older persons: the cardiovascular health study. Arch Intern Med. 2006;166:23882394.

23. Jeganathan VS, Kawasaki R, Wang JJ, et al. Retinal vascular caliber and age-related macular degeneration: the Singapore Malay Eye Study. Am J Opbthalmol. 2008;146:954-959.

24. Sun C, Liew G, Wang JJ, et al. Retinal vascular caliber, blood pressure, and cardiovascular risk factors in an Asian population: the Singapore Malay Eye Study. Invest Ophthalmol Vis Sci. 2008; 49:1784-1790.

25. McGeechan K, Liew G, Macaskill P, et al. Prediction of incident stroke events based on retinal vessel caliber: a systematic review and individual-participant meta-analysis. Am J Epidemiol. 2009; 170:1323-1332.

26. McGeechan K, Liew G, Macaskill P, et al. Meta-analysis: retinal vessel caliber and risk for coronary heart disease. Ann Intern Med. 2009; $151: 404-413$.

27. Tolentino MJ, McLeod DS, Taomoto M, Otsuji T, Adamis AP, Lutty GA. Pathologic features of vascular endothelial growth factorinduced retinopathy in the nonhuman primate. Am J Opbthalmol. 2002;133:373-385.

28. Bengtsson B, Krakau CE. Correction of optic disc measurements on fundus photographs. Graefes Arch Clin Exp Opbthalmol. 1992; 230:24-28. 